\title{
NIELSEN COINCIDENCE, FIXED POINT AND ROOT THEORIES OF $n$-VALUED MAPS
}

\author{
Robert F. Brown \\ Department of Mathematics \\ University of California \\ Los Angeles, CA 90095-1555 \\ e-mail: rfb@math.ucla.edu \\ Kameran Kolahi \\ Department of Mathematics \\ University of California \\ Los Angeles, CA 90095-1555 \\ e-mail: kkolahi@ucla.edu
}

April 17, 2013

\begin{abstract}
Let $(\phi, \psi)$ be an $(m, n)$-valued pair of maps $\phi, \psi: X \multimap Y$, where $\phi$ is an $m$-valued map and $\psi$ is $n$-valued, on connected finite polyhedra. A point $x \in X$ is a coincidence point of $\phi$ and $\psi$ if $\phi(x) \cap \psi(x) \neq \emptyset$. We define a Nielsen coincidence number $N(\phi: \psi)$ which is a lower bound for the number of coincidence points of all $(m, n)$-valued pairs of maps homotopic to $(\phi, \psi)$. We calculate $N(\phi: \psi)$ for all $(m, n)$-valued pairs of maps of the circle and show that $N(\phi: \psi)$ is a sharp lower bound in that setting. Specifically, if $\phi$ is of degree $a$ and $\psi$ of degree $b$, then

$$
N(\phi: \psi)=\frac{|a n-b m|}{<m, n>}
$$

where $\langle m, n\rangle$ is the greatest common divisor of $m$ and $n$. In order to carry out the calculation, we obtain results, of independent interest, for $n$ valued maps of compact connected Lie groups that relate the Nielsen fixed point number of Helga Schirmer to the Nielsen root number of Michael Brown.
\end{abstract}

Subject Classification 55M20, 54C60

\section{Introduction}

A multivalued function $\phi: X \multimap Y$ is continuous if it is both upper and lower semi-continuous. A function $\phi$ is $n$-valued if $\phi(x)$ is 
an unordered set of $n$ points of $Y$ for each $x \in X$. As in [10], a continuous $n$-valued function is called an $n$-valued map.

For a multivalued function $\phi: X \multimap X$, a point $x \in X$ is a fixed point of $\phi$ if $x \in \phi(x)$. The fixed point concept may be generalized as follows: let $\phi, \psi: X \multimap Y$ be multivalued functions, then $x \in X$ is a coincidence point of $\phi$ and $\psi$ if $\phi(x) \cap \psi(x) \neq \emptyset .^{1}$ Define the coincidence set $\operatorname{Coin}(\phi: \psi)$ of $\phi$ and $\psi$ by

$$
\operatorname{Coin}(\phi: \psi)=\{x \in X: \phi(x) \cap \psi(x) \neq \emptyset\} .
$$

If $\phi, \psi: X \multimap Y$, where $\phi$ is an $m$-valued map and $\psi$ is an $n$ valued map, then we call the pair $(\phi, \psi)$ an $(m, n)$-valued pair of maps. An $n$-valued homotopy is an $n$-valued map $\Phi: X \times I \multimap Y$, and we say that the $n$-valued maps $\phi_{0}$ and $\phi_{1}$, where $\phi_{t}(x)=\Phi(x, t)$, are (n-valued) homotopic. Then $(m, n)$-valued pairs of maps $(\phi, \psi)$ and $\left(\phi^{\prime}, \psi^{\prime}\right)$ are $((m, n)$-valued $)$ homotopic if there is an $m$-valued homotopy $\Phi: X \times I \multimap Y$ and an $n$-valued homotopy $\Psi: X \times I \multimap Y$ such that $\phi_{0}=\phi, \phi_{1}=\phi^{\prime}, \psi_{0}=\psi$ and $\psi_{1}=\psi^{\prime}$. The minimum number for coincidences $M C(\phi: \psi)$ of an $(m, n)$-valued pair of maps $(\phi, \psi)$ is defined to be the minimum cardinality of $\operatorname{Coin}\left(\phi^{\prime}, \psi^{\prime}\right)$ among all $(m, n)$-valued pairs of maps $\left(\phi^{\prime}, \psi^{\prime}\right)$ homotopic to $(\phi, \psi)$.

The Nielsen fixed point theory of $n$-valued maps $\phi: X \multimap X$, where $X$ is a connected finite polyhedron, was introduced by Schirmer in [17] - [19] and has been developed further in [1] - [2], [6] - [10]. There is an extensive Nielsen coincidence theory for single-valued maps that is described in the survey of Goncalves [13]. In this paper, we present the Nielsen coincidence theory for $(m, n)$-valued pairs of maps. The paper [7] calculated the Nielsen fixed point numbers of all $n$-valued maps of the circle and showed that, in that setting, the Nielsen number calculates the minimum fixed point number. We will here determine the Nielsen coincidence theory of $(m, n)$-valued pairs of maps of the circle by calculating the Nielsen coincidence numbers of all such pairs, and we will demonstrate that it equals the minimum coincidence number.

In Section 2, we extend the definition of the Nielsen coincidence number for single-valued maps, due to Brooks (see for instance [3]), to define the Nielsen coincidence number $N(\phi: \psi)$ of an $(m, n)$ valued pair of maps $\phi, \psi: X \multimap Y$. We show that $N(\phi: \psi)$ is a homotopy invariant and therefore $N(\phi: \psi) \leq M C(\phi: \psi)$. We also describe in this section the Nielsen root theory of Michael Brown

\footnotetext{
${ }^{1}$ Although this definition of "coincidence" is that of [12], for instance, it is not the only one in the setting of multivalued functions (see [21]). Much of the literature concerns the special case in which $\phi=f$ is single-valued and "coincidence" is defined to mean $f(x) \in \psi(x)$, and that does correspond to the definition that we use.
} 
[6]. Let $\phi: X \multimap Y$ be an $n$-valued map and let $a \in Y$. Then $x \in X$ is a root of $\phi$ at $a$ if $a \in \phi(x)$; write $x \in \phi^{-1}(a)$. The Nielsen root number $N(\phi, a)$, introduced in [6], is a lower bound for the number of points in $\psi^{-1}(a)$ for all $n$-valued maps $\psi$ homotopic to $\phi$.

Section 3 is concerned with the fixed point theory of $n$-valued maps. We relate Schirmer's theory in [18], based on algebraic topology, to a more geometric theory that is similar to the coincidence theory of Section 2.

In order to study the coincidence theory of $(m, n)$-valued pairs of maps of the circle, we first devote Section 4 to a more general topic of independent interest. Let $X$ be a compact connected Lie group with identity element $e$ and let $\phi, \psi: X \multimap X$ be an $(m, n)$-valued pair of maps. If $\phi(x)=\left\{w_{1}, \ldots, w_{m}\right\}$ and $\psi(x)=\left\{z_{1}, \ldots, z_{n}\right\}$, let $\phi \psi^{-1}(x)=\left\{w_{i} z_{j}^{-1}: i=1, \ldots, m ; j=1, \ldots, n\right\}$. If the number of distinct points of $X$ in the set $\phi \psi^{-1}(x)$ is the same number $u$ of points for all $x \in X$, then there is a $u$-valued map $\theta: X \multimap X$ defined by letting $\theta(x)$ be the unordered set of distinct points in $\phi \psi^{-1}(x)$. If $\psi: X \rightarrow X$ is the identity map, then for $\phi(x)=\left\{x_{1}, \ldots, x_{m}\right\}$, we have $\theta(x)=\left\{x_{1} x^{-1}, \ldots, x_{m} x^{-1}\right\}$. Then $x$ is a fixed point of $\phi$ if and only if it is a root of $\theta$ at $e$. The corresponding Nielsen theories agree, that is, $N(\theta, e)=N(\phi)$, the Nielsen root number equals Schirmer's Nielsen fixed point number of $\phi$ [18] (except, possibly, in the case that $X$ is the 2 -torus).

Section 5 specializes the previous results to $n$-valued maps of the circle $S^{1}$. Since the Nielsen fixed point numbers of $n$-valued maps of $S^{1}$ were calculated in [7], we can use the result of Section 4 to calculate the Nielsen root numbers of such maps. By [8], an $n$-valued map of $S^{1}$ induces a (single-valued) endomorphism of $H_{1}\left(S^{1}\right)$, so the degree $\operatorname{deg}(\phi)$ is defined as for single-valued maps. We prove that the Nielsen root number of an $n$-valued map $\phi: S^{1} \multimap S^{1}$ is $N(\phi, 1)=|\operatorname{deg}(\phi)|$. We then consider the Nielsen coincidence theory of $(m, n)$-valued pairs of maps $\phi, \psi: S^{1} \multimap S^{1}$. Let $\left.<m, n\right\rangle$ denote the greatest common divisor of $m$ and $n$. We prove that if the $m$ valued map $\phi$ is of degree $a$ and the $n$-valued map $\psi$ is of degree $b$, then there is $u$-valued map $\theta: S^{1} \multimap S^{1}$ of degree $\delta$, where

$$
u=\frac{m n}{<m, n\rangle}, \quad \delta=\frac{a n-b m}{<m, n\rangle},
$$

such that $N(\phi: \psi)=N(\theta, 1)$ and therefore

$$
N(\phi: \psi)=\frac{|a n-b m|}{<m, n>} .
$$

We use this calculation to prove that $N(\phi: \psi)=M C(\phi: \psi)$ for all 
$(m, n)$-valued pairs of maps of the circle to itself.

\section{The Nielsen coincidence and root numbers}

For an $n$-valued map $\phi: X \multimap Y$ of compact connected metric spaces, if $\phi(x)=\left\{y_{1}, \ldots, y_{n}\right\}$ we let $\gamma(x, \phi)$ denote the minimum of the distances among the $y_{j}$. The gap [17] of $\phi$, denoted $\gamma(\phi)$, is the infimum of the $\gamma(x, \phi)$ for all $x \in X$. The compactness of $X$ implies that $\gamma(\phi)>0$.

The Splitting Lemma (see, for instance, Lemma 1 on page 74 of [17]) states that if $\phi: X \multimap Y$ is an $n$-valued map of connected finite polyherdra and $X$ is simply-connected, then $\phi$ is split. That is, there exist (single-valued) maps $f_{1}, \ldots, f_{n}: X \rightarrow Y$ such that $\phi(x)=\left\{f_{1}(x), \ldots, f_{n}(x)\right\}$ for all $x \in X$.

Proposition 2.1. Let $\phi: X \multimap Y$ be an n-valued map, where $X$ and $Y$ are compact connected metric spaces. If $\phi$ is split, then the splitting is unique, that is, if $\phi(x)=\left\{f_{1}(x), \ldots, f_{n}(x)\right\}=\left\{g_{1}(x), \ldots, g_{n}(x)\right\}$ for all $x \in X$, then $\left\{f_{1}, \ldots, f_{n}\right\}=\left\{g_{1}, \ldots, g_{n}\right\}$ as unordered sets of single-valued functions. ${ }^{2}$

Proof. Let $x_{0} \in X$ and write $\phi\left(x_{0}\right)=\left\{y_{1}, \ldots, y_{n}\right\}$. We may assume that $y_{1}=f_{1}\left(x_{0}\right)=g_{1}\left(x_{0}\right)$, and it is sufficient to prove that $f_{1}(x)=$ $g_{1}(x)$ for all $x \in X$. Denote the metrics of $X$ and $Y$ by $d_{X}$ and $d_{Y}$. Let $\epsilon>0$ be such that $\epsilon<\gamma(\phi) / 2$, where $\gamma(\phi)$ is the gap of $\phi$. Since $f_{1}$ and $g_{1}$ are continuous, there exists $\delta>0$ such that $d_{X}\left(x, x_{0}\right)<\delta$ implies $d_{Y}\left(f_{1}(x), f_{1}\left(x_{0}\right)\right)<\epsilon$ and $d_{Y}\left(g_{1}(x), g_{1}\left(x_{0}\right)\right)<\epsilon$. Suppose that $d_{X}\left(x, x_{0}\right)<\delta$ and $f_{1}(x)=g_{j}(x)$ for some $j \neq 1$. Then

$$
\begin{aligned}
d_{Y}\left(g_{j}(x), g_{1}(x)\right) & =d_{Y}\left(f_{1}(x), g_{1}(x)\right) \\
& \leq d_{Y}\left(f_{1}(x), f_{1}\left(x_{0}\right)\right)+d_{Y}\left(f_{1}\left(x_{0}\right), g_{1}(x)\right) \\
& =d_{Y}\left(f_{1}(x), f_{1}\left(x_{0}\right)\right)+d_{Y}\left(g_{1}\left(x_{0}\right), g_{1}(x)\right) \\
& <2 \epsilon<\gamma(\phi),
\end{aligned}
$$

which contradicts the definition of the gap $\gamma(\phi)$, and therefore $f_{1}(x)=$ $g_{1}(x)$ for all $x$ in a $\delta$-neighborhood. Thus, the subset $\operatorname{Coin}\left(f_{1}, g_{1}\right)$ of $X$ on which $f_{1}=g_{1}$ is open. But the same argument proves that $\operatorname{Coin}\left(f_{1}, g_{j}\right)$ is open for all $g_{j}$ with $j \neq 1$, so $\operatorname{Coin}\left(f_{1}, g_{1}\right)$ is also closed. Since $X$ is connected, we conclude that $\operatorname{Coin}\left(f_{1}, g_{1}\right)=X$, that is, $f_{1}(x)=g_{1}(x)$ for all $x \in X$.

\footnotetext{
${ }^{2}$ This simple observation has not appeared in the literature, and since [18] refers more than once to "a splitting" rather than "the splitting", we include it here to prevent any misunderstanding.
} 
Let $\phi, \psi: X \multimap Y$ be an $(m, n)$-valued pair of maps of connected finite polyhedra. Define an equivalence relation on $\operatorname{Coin}(\phi: \psi)$ by calling $x$ and $x^{\prime}$ equivalent coincidence points if there is a path $p: I \rightarrow X$ such that $p(0)=x, p(1)=x^{\prime}$ and $p$ has the following property: For the splittings $\phi p=\left\{f_{1}, \ldots, f_{m}\right\}$ and $\psi p=\left\{g_{1}, \ldots, g_{n}\right\}$, there exist $1 \leq j \leq m$ and $1 \leq k \leq n$ such that $f_{j}(0)=g_{k}(0)$, $f_{j}(1)=g_{k}(1)$ and the paths $f_{j}, g_{k}: I \rightarrow Y$ are homotopic relative to the end points. The corresponding equivalence classes are called the coincidence classes of $\phi$ and $\psi$, which are finite in number because $X$ is compact.

The next result corresponds to Lemmas 6.2 and 6.3 of [18] in the setting of coincidences of $(m, n)$-valued pairs of maps. For $\Phi: X \times$ $I \multimap Y$ an $n$-valued homotopy, let $\phi_{t}: X \times\{t\} \multimap Y$ be the restriction of $\Phi$.

Lemma 2.1. Let $\Phi, \Psi: X \times I \multimap Y$ be an $(m, n)$-valued pair of homotopies. The intersection $\mathbf{C}_{t}$ of a coincidence class $\mathbf{C}$ of $\Phi$ and $\Psi$ with $X \times\{t\}$ is either empty or a coincidence class of $\phi_{t}$ and $\psi_{t}$. Each coincidence class of $\phi_{t}$ and $\psi_{t}$ is contained in a unique coincidence class of $\Phi$ and $\Psi$.

Proof. Suppose $(x, t)$ and $\left(x^{\prime}, t\right)$ are in a coincidence class $\mathbf{C}$ of $\Phi$ and $\Psi$. Then there is a path $p: I \rightarrow X \times I$ such that $p(0)=$ $(x, t), p(1)=\left(x^{\prime}, t\right)$ and from the splittings $\Phi p=\left\{f_{1}, \ldots, f_{m}\right\}$ and $\Psi p=\left\{g_{1}, \ldots, g_{n}\right\}$, we may order the maps so that $f_{1}(0)=g_{1}(0) \in$ $\Phi(x, t) \cap \Psi(x, t), f_{1}(1)=g_{1}(1) \in \Phi\left(x^{\prime}, t\right) \cap \Psi\left(x^{\prime}, t\right)$ and $f_{1}$ and $g_{1}$ are homotopic in $Y$ relative to the endpoints. Let $\pi_{t}: X \times I \rightarrow$ $X \times\{t\}$ be the projection map and define $\bar{p}=\pi_{t} p: I \rightarrow X \times\{t\}$. Then there is a homotopy $H: I \times I \rightarrow X$ between $\bar{p}$ and $p$. The splittings of $\Phi H$ and $\Psi H$ restrict to $\Phi \bar{p}=\phi_{t} \bar{p}=\left\{\bar{f}_{1}, \ldots, \bar{f}_{m}\right\}$ and $\Psi \bar{p}=\psi_{t} \bar{p}=\left\{\bar{g}_{1}, \ldots, \bar{g}_{n}\right\}$, where, in particular, $\bar{f}_{1}$ is homotopic to $f_{1}$ and $\bar{g}_{1}$ is homotopic to $g_{1}$. Since the homotopy $H$ is constant at the endpoints, $\bar{f}_{1}(0)=\bar{g}_{1}(0)$ and $\bar{f}_{1}(1)=\bar{g}_{1}(1)$. Combining homotopies, we obtain a homotopy $K: I \times I \rightarrow Y$ between $\bar{f}_{1}$ and $\bar{g}_{1}$ relative to the endpoints that establishes the fact that $(x, t)$ and $\left(x^{\prime}, t\right)$ are in the same coincidence class of $\phi_{t}$ and $\psi_{t}$. We have proved that the nonempty intersection $\mathbf{C}_{t}$ of $\mathbf{C}$ and $X \times\{t\}$ is a coincidence class of $\phi_{t}$ and $\psi_{t}$. Conversely, suppose that $(x, t)$ and $\left(x^{\prime}, t\right)$ are in a coincidence class $\mathbf{C}_{t}$ of $\phi_{t}$ and $\psi_{t}$. A path $p: I \rightarrow X \times\{t\}$ that demonstrates their equivalence as coincidence points of $\phi_{t}$ and $\psi_{t}$ also shows that they are equivalent coincidence points of $\Phi$ and $\Psi$. Thus, they are in a coincidence class $\mathbf{C}$ such that $\mathbf{C} \cap(X \times\{t\})=$ $\mathbf{C}_{t}$. 
A coincidence class $\mathbf{C}_{0}$ of an $(m, n)$-valued pair of maps $\phi, \psi: X \multimap$ $Y$ is inessential if there are homotopies $\Phi, \Psi: X \times I \multimap Y$ such that $\phi_{0}=\phi, \psi_{0}=\psi$ and the coincidence class $\mathbf{C}$ of $\Phi$ and $\Psi$ containing $\mathbf{C}_{0}$ has the property $\mathbf{C} \cap(X \times\{1\})=\emptyset$. Otherwise, the coincidence class is essential. The Nielsen coincidence number $N(\phi: \psi)$ is the number of essential coincidence classes. ${ }^{3}$

Theorem 2.1. The Nielsen coincidence number for $(m, n)$-valued pairs of maps of connected finite polyhedra is a homotopy invariant. That is, if $\phi, \phi^{\prime}: X \multimap Y$ and $\psi, \psi^{\prime}: X \multimap Y$ are homotopic respectively, then $N(\phi: \psi)=N\left(\phi^{\prime}: \psi^{\prime}\right)$. Therefore, $N(\phi: \psi) \leq M C(\phi$ : $\psi)$.

Proof. Let $\Phi, \Psi: X \times I \multimap Y$ be homotopies such that $\phi_{0}=\phi, \phi_{1}=$ $\phi^{\prime}, \psi_{0}=\psi$ and $\psi_{1}=\psi^{\prime}$. Let $\mathbf{C}_{0}$ be an essential coincidence class of $\phi$ and $\psi$ and let $\mathbf{C}$ be the coincidence class of $\Phi$ and $\Psi$ containing $\mathbf{C}_{0}$. Then $\mathbf{C}_{1}=\mathbf{C} \cap(X \times\{1\})$ is a coincidence class of $\phi^{\prime}$ and $\psi^{\prime}$. If $\mathbf{C}_{1}$ was an inessential coincidence class of $\phi^{\prime}$ and $\psi^{\prime}$, then there would be an $(m, n)$-valued pair of homotopies $\Phi^{\prime}, \Psi^{\prime}: X \times I \multimap Y$ such that for $\mathbf{C}^{\prime}$, the coincidence class of $\Phi^{\prime}$ and $\Psi^{\prime}$ containing $\mathbf{C}_{1}$, we would have $\mathbf{C}^{\prime} \cap(X \times\{1\})=\emptyset$. Define homotopies $\Phi^{\prime \prime}, \Psi^{\prime \prime}: X \times I \multimap Y$ by

$$
\Phi^{\prime \prime}(x, t)= \begin{cases}\Phi(x, 2 t) & \text { if } 0 \leq t \leq \frac{1}{2} \\ \Phi^{\prime}(x, 1-2 t) & \text { if } \frac{1}{2} \leq t \leq 1\end{cases}
$$

and $\Psi^{\prime \prime}$ similarly. The set $\mathbf{C}^{\prime \prime}=\mathbf{C} \cup \mathbf{C}^{\prime}$ would be a coincidence class of $\Phi^{\prime \prime}, \Psi^{\prime \prime}$ containing $\mathbf{C}$, an essential coincidence class, but $\mathbf{C}^{\prime \prime} \cap(X \times\{1\})=\emptyset$. Therefore, $\mathbf{C}_{1}$ is also essential and the pair $(\Phi, \Psi)$ determines a one-to-one correspondence between the essential coincidence classes of $\phi$ and $\psi$ and the essential coincidence classes of $\phi^{\prime}$ and $\psi^{\prime}$. In the same way, the pair of $(m, n)$-valued homotopies $\bar{\Phi}, \bar{\Psi}: X \times I \multimap Y$ defined by $\bar{\Phi}(x, t)=\Phi(x, 1-t)$ and $\bar{\Psi}(x, t)=\Psi(x, 1-t)$ determine a one-to-one correspondence between the essential coincidence classes of $\phi^{\prime}$ and $\psi^{\prime}$ and the essential coincidence classes of $\phi$ and $\psi$, so $N(\phi: \psi)=N\left(\phi^{\prime}: \psi^{\prime}\right)$. Since each $(m, n)$-valued pair $\left(\phi^{\prime}, \psi^{\prime}\right)$ homotopic to $(\phi, \psi)$ therefore has at least $N(\phi: \psi)$ coincidence classes, then $N(\phi: \psi) \leq M C(\phi: \psi)$.

Let $\phi: X \multimap Y$ be an $n$-valued map. For $a \in Y$, a point $x \in X$ is called a root of $\phi$ at $a$ if $a \in \phi(x)$. We denote the set of roots of $\phi$ at $a$ by $\phi^{-1}(a)$. Following [6], we define an equivalence relation on $\phi^{-1}(a)$ by calling $x_{0}, x_{1}$ equivalent if there is a map $p: I \rightarrow X$ such that $p(0)=x_{0}, p(1)=x_{1}$ and for the splitting $\phi p=\left\{f_{1}, \ldots, f_{n}\right\}$,

\footnotetext{
${ }^{3}$ If $m=n=1$, this definition agrees with that of the $\Delta$-Nielsen number of [5].
} 
there is $f_{i}: I \rightarrow Y$ that is a nullhomotopic loop at $a$. That is, there is a map $H: I \times I \rightarrow Y$ such that $H(s, 0)=f_{i}(s), H(s, 1)=a$ for all $s \in I$ and $H(0, t)=H(1, t)=a$ for all $t \in I$. An equivalence class $\mathbf{R}$ is called a root class of $\phi$ at $a$.

¿From Lemma 2.1, in the case that $\Psi: X \times I \rightarrow Y$ is the constant map at $a$, we obtain:

Lemma 2.2. Let $\Phi: X \times I \multimap Y$ be an n-valued homotopy and let $a \in Y$. The intersection $\mathbf{R}_{t}$ of a root class $\mathbf{R}$ of $\Phi$ at a with $X \times\{t\}$ is either empty or a root class of $\phi_{t}$ at a. Consequently, each root class $\mathbf{R}_{t}$ of $\phi_{t}$ at a is contained in a unique root class of $\Phi$ at a.

Let $\phi: X \multimap Y$ be an $n$-valued map and let $a \in Y$. A root class $\mathbf{R}_{0}$ of $\phi$ at $a$ is inessential if there is a homotopy $\Phi: X \times I \multimap Y$ such that $\phi_{0}=\phi$ and $\mathbf{R}$, the root class of $\Phi$ at $a$ that contains $\mathbf{R}_{0}$, has the property $\mathbf{R} \cap(X \times\{1\})=\emptyset$. Otherwise, $\mathbf{R}_{0}$ is essential. The Nielsen root number $N(\phi, a)$ of $\phi$ at $a$ is the number of essential root classes. ${ }^{4}$

\section{$3 \quad$ Nielsen fixed point theories}

We next review the Nielsen fixed point theory of Schirmer [18]. Given an $n$-valued map $\phi: X \multimap X$, where $X$ is a connected finite polyhedron, define an equivalence relation on

$$
F i x(\phi)=\{x \in X: x \in \phi(x)\}
$$

by calling $x, x^{\prime} \in F i x(\phi)$ equivalent if there exists a path $p: I \rightarrow X$ such that $p(0)=x, p(1)=x^{\prime}$ and, in the splitting $\phi p=\left\{f_{1}, \ldots, f_{n}\right\}$, some $f_{j}: I \rightarrow X$ has the properties $f_{j}(0)=x, f_{j}(1)=x^{\prime}$ and $f_{j}$ is homotopic to $p$ relative to the endpoints. An equivalance class $\mathbf{F}$ is called a fixed point class. Thus, the definition of a fixed point class can be viewed as the special case of a coincidence class for an $(m, 1)$-valued pair of maps $\phi, \psi: X \multimap X$, where $\psi: X \rightarrow X$ is the identity map.

In [18], Schirmer developed an integer-valued fixed point index theory for $n$-valued maps and called a fixed point class $\mathbf{F}$ essential if its index $\operatorname{ind}(\mathbf{F})$ is nonzero. We shall say that a class $\mathbf{F}$ is algebraically essential if ind $(\mathbf{F}) \neq 0$ and algebraically inessential otherwise. Schirmer's Nielsen fixed point number $N(\phi)$ is the number of algebraically essential fixed point classes.

\footnotetext{
${ }^{4}$ The definition of an inessential root class, and thus of the Nielsen root number, in [6] is in terms of a generalization of the Hopf covering space (see [4], Definition 3.3). However, it follows from Theorem 3.1 of [9] that the definition in [6] is equivalent to the one given here.
} 
Let $\Phi: X \times I \multimap X$ be an $n$-valued homotopy. The corresponding fat homotopy (see [15], Def. 6.1) $\widehat{\Phi}: X \times I \multimap X \times I$ is defined as follows: if $\Phi(x, t)=\left\{x_{1}, \ldots, x_{n}\right\}$, then $\widehat{\Phi}(x, t)=\left\{\left(x_{1}, t\right), \ldots,\left(x_{n}, t\right)\right\}$. If we apply Lemma 2.1 to the case of $Y=X \times I$ and $\Psi$ the identity map of $X \times I$, we conclude that the intersection $\widehat{\mathbf{F}}_{t}$ of a fixed point class $\widehat{\mathbf{F}}$ of the fat homotopy $\widehat{\Phi}$ and $X \times\{t\}$ is either empty or a fixed point class of $\phi_{t}$. Consequently, each fixed point class $\mathbf{F}$ of $\phi_{t}$ is contained in a unique fixed point class $\widehat{\mathbf{F}}$ of the fat homotopy $\widehat{\Phi}$. We call a fixed point class $\mathbf{F}$ of an $n$-valued map $\phi: X \multimap X$ geometrically inessential if there is a homotopy $\Phi: X \times I \multimap X$ such that $\phi_{0}=\phi$ and the fixed point class $\widehat{\mathbf{F}}$ of the fat homotopy $\widehat{\Phi}$ such that $\widehat{\mathbf{F}}_{0}=\widehat{\mathbf{F}} \cap(X \times\{0\})=\mathbf{F}$ has the property $\widehat{\mathbf{F}}_{1}=\widehat{\mathbf{F}} \cap(X \times\{1\})=\emptyset$. Otherwise, $\mathbf{F}$ is geometrically essential. We define the geometric Nielsen fixed point number $\widehat{N}(\phi)$ of an $n$-valued map $\phi: X \multimap X$ of a finite polyhedron to be the number of geometrically essential fixed point classes of $\phi$. Since $\widehat{N}(\phi)=N(\phi: \psi)$, where $\psi$ is the identity map of $X$, it follows from Theorem 2.1 that $\widehat{N}(\phi)$ is a homotopy invariant.

A point $x$ in a space $X$ is a local cut point if there is a connected neighborhood $U$ of $x$ such that $U \backslash x$ is disconnected.

Proposition 3.1. If $X=S^{1}$ or if $X$ is a connected finite polyhedron without local cut points that is not a 2-manifold, then $\widehat{N}(\phi)=N(\phi)$.

Proof. Let $\mathbf{F}$ be an algebraically essential fixed point class of $\phi$ and let $\Phi: X \times I \multimap X$ be a homotopy such that $\phi_{0}=\phi$. Let $\widehat{\mathbf{F}}$ be the fixed point class of the fat homotopy $\widehat{\Phi}$ such that $\widehat{\mathbf{F}}_{0}=\mathbf{F}$. Then $\operatorname{ind}\left(\widehat{\mathbf{F}}_{0}\right) \neq 0$. By Lemma 6.4 of $[18]$, ind $\left(\widehat{\mathbf{F}}_{0}\right)=\operatorname{ind}\left(\widehat{\mathbf{F}}_{1}\right)$, so $\widehat{\mathbf{F}}_{1} \neq \emptyset$ by Corollary 4.7 of [18] and therefore, $\mathbf{F}$ is geometrically essential. To prove the converse, suppose $\operatorname{ind}(\mathbf{F})=0$. By Theorem 5.1 of [7] if $X=S^{1}$ or by [2] otherwise, there is a homotopy $\Phi: X \times I \multimap X$ such that $\phi_{0}=\phi$ and $\phi_{1}$ has exactly $N(\phi)$ fixed points. Thus, each fixed point class of $\phi_{1}$ consists of a single point of nonzero index. Let $\widehat{\mathbf{F}}$ be the fixed point class of the fat homotopy $\widehat{\Phi}$ corresponding to the homotopy of Theorem 5.1 of [7] or that of [2] such that $\widehat{\mathbf{F}}_{0}=$ $\mathbf{F}$. If $\widehat{\mathbf{F}}_{1}$ were nonempty, by Lemma 6.4 of [18], $\widehat{\mathbf{F}}_{1}$ would be an algebraically inessential fixed point class of $\phi_{1}$. But since $\phi_{1}$ has no such fixed point class, we conclude that $\widehat{\mathbf{F}}_{1}=\emptyset$ and therefore, $\mathbf{F}$ is geometrically inessential.

Proposition 3.1 is false if $X$ is a hyperbolic surface. The single fixed point class of the map $f$ in the examples of [14] is algebraically inessential but geometrically essential, so $N(f)=0$ but $\widehat{N}(f)=1$. 
The proposition is true for single-valued maps of other surfaces, but it is not known whether $\widehat{N}(\phi)=N(\phi)$ for $\phi$ an $n$-valued map of a non-hyperbolic surface with $n>1$.

\section{Nielsen theories on Lie groups}

Let $\phi, \psi: W \multimap X$ be an $(m, n)$-valued pair of maps, where $W$ is a connected finite polyhedron and $X$ is a compact connected Lie group with identity element $e$. For each $w \in W$, define $\phi \psi^{-1}(w)$ as follows: if we write $\phi(w)=\left\{y_{1}, \ldots, y_{m}\right\}$ and $\psi(w)=\left\{z_{1}, \ldots, z_{n}\right\}$, then

$$
\phi \psi^{-1}(w)=\left\{y_{i} z_{j}^{-1}: i=1, \ldots, m ; j=1, \ldots, n\right\} .
$$

Therefore, $\phi \psi^{-1}(w)$ is a point in the configuration space $C_{m n}(X)$ of unordered subsets of $m n$ not necessarily distinct points of $X$. The function $\phi \psi^{-1}: W \rightarrow C_{m n}(X)$ defined in this way is continuous. In general, the number of distinct points of $X$ in the set $\phi \psi^{-1}(w)$ will vary with $w$. However, if the number $u$ of distinct points in $\phi \psi^{-1}(w)$ is the same for all $w \in W$, we may define a $u$-valued map $\theta: W \multimap X$ by letting $\theta(w)$ be the unordered set of the distinct points of $\phi \psi^{-1}(w)$. We will call $\theta$ the $u$-valued map determined by $\phi$ and $\psi$. We note that $w$ is a coincidence point of $\phi$ and $\psi$ if and only if it is a root of $\theta$ at $e$, that is, $\operatorname{Coin}(\phi: \psi)=\theta^{-1}(e)$.

Lemma 4.1. Let $\phi, \psi: W \multimap X$ be an $(m, n)$-valued pair of maps, where $W$ is a connected finite polyhedron and $X$ is a compact connected Lie group, such that $\phi$ and $\psi$ determine a u-valued map $\theta: W \multimap X$. Let $v: Z \rightarrow W$ be a single-valued map, where $Z$ is a simply-connected finite polyhedron. Then for the splittings $\phi v=$ $\left\{f_{1}, \ldots, f_{m}\right\}, \psi v=\left\{g_{1}, \ldots, g_{n}\right\}$ and $\theta v=\left\{h_{1}, \ldots, h_{u}\right\}$, for each $i=1, \ldots$, u there exist $1 \leq j_{i} \leq m, 1 \leq k_{i} \leq n$ such that $h_{i}=f_{j_{i}} g_{k_{i}}^{-1}$.

Proof. To simplify notation, we denote by $d$ the metrics of both $Z$ and $X$. Since the maps $f_{j}, g_{k}: Z \rightarrow X$ and the group operations are continuous, there exists $\delta>0$ such that if $d\left(z, z^{\prime}\right)<\delta$, then

$$
d\left(f_{j}(z) g_{k}(z)^{-1}, f_{j}\left(z^{\prime}\right) g_{k}\left(z^{\prime}\right)^{-1}\right)<\frac{\gamma(\theta)}{3}
$$

for all $j$ and $k$, where $\gamma(\theta)$ is the gap of $\theta$. Let $1 \leq j, j^{\prime} \leq m$ with $j \neq j^{\prime}$ and $1 \leq k, k^{\prime} \leq n$ with $k \neq k^{\prime}$ and define

$$
\begin{aligned}
& A=\left\{z \in Z: f_{j}(z) g_{k}(z)^{-1} \neq f_{j^{\prime}}(z) g_{k^{\prime}}(z)^{-1}\right\} \\
& B=\left\{z \in Z: f_{j}(z) g_{k}(z)^{-1}=f_{j^{\prime}}(z) g_{k^{\prime}}(z)^{-1}\right\} .
\end{aligned}
$$


If $z \in A$ and $d\left(z, z^{\prime}\right)<\delta$, then $z^{\prime} \in A$ because

$$
d\left(f_{j}\left(z^{\prime}\right) g_{k}\left(z^{\prime}\right)^{-1}, f_{j^{\prime}}\left(z^{\prime}\right) g_{k^{\prime}}\left(z^{\prime}\right)^{-1}\right)>\frac{\gamma(\theta)}{3} .
$$

If $z \in B$ and $d\left(z, z^{\prime}\right)<\delta$, then

$$
d\left(f_{j}\left(z^{\prime}\right) g_{k}\left(z^{\prime}\right)^{-1}, f_{j^{\prime}}\left(z^{\prime}\right) g_{k^{\prime}}\left(z^{\prime}\right)^{-1}\right)<\frac{2 \gamma(\theta)}{3} .
$$

But $f_{j}\left(z^{\prime}\right) g_{k}\left(z^{\prime}\right)^{-1}$ and $f_{j^{\prime}}\left(z^{\prime}\right) g_{k^{\prime}}\left(z^{\prime}\right)^{-1}$ are in $\theta\left(z^{\prime}\right)$, so then they must be equal and therefore $z^{\prime} \in B$. We have proved that both $A$ and $B$ are open in the connected space $Z$, and thus one of them must be empty for any given $j, k, j^{\prime}$ and $k^{\prime}$. So choose a basepoint $z_{0} \in Z$ and write the distinct points of $\phi \psi^{-1}\left(z_{0}\right)$ as

$$
\left\{f_{j_{1}}\left(z_{0}\right) g_{k_{1}}\left(z_{0}\right)^{-1}, \ldots, f_{j_{u}}\left(z_{0}\right) g_{k_{u}}\left(z_{0}\right)^{-1}\right\} \text {. }
$$

Since for all $1 \leq i, i^{\prime} \leq u$ with $i \neq i^{\prime}$ and all $z \in Z$ we have $f_{j_{i}}(z) g_{k_{i}}(z)^{-1} \neq f_{j_{i^{\prime}}}(z) g_{k_{i^{\prime}}}(z)^{-1}$, we conclude that

$$
\theta v=\left\{f_{j_{1}} g_{k_{1}}^{-1}, \ldots, f_{j_{u}} g_{k_{u}}^{-1}\right\}
$$

is the splitting of $\theta v$.

Theorem 4.1. Let $\phi, \psi: W \multimap X$ be an $(m, n)$-valued pair of maps, where $W$ is a connected finite polyhedron and $X$ is a compact connected Lie group with identity element $e$, such that $\phi$ and $\psi$ determine the u-valued map $\theta: W \multimap X$. Then $x_{0}$ and $x_{1}$ are equivalent as coincidence points of $\phi$ and $\psi$ if and only if they are equivalent roots of $\theta$ at $e$. Therefore, the coincidence classes of $\phi$ and $\psi$ are the root classes of $\theta$ at $e$.

Proof. Let $p: I \rightarrow W$ be a path, then there are splittings $\phi p=$ $\left\{f_{1}, \ldots, f_{m}\right\}$ and $\psi p=\left\{g_{1}, \ldots, g_{n}\right\}$ and, by Lemma 4.1,

$$
\theta p=\left\{h_{1}, \ldots, h_{u}\right\}=\left\{f_{j_{1}} g_{k_{1}}^{-1}, \ldots, f_{j_{u}} g_{k_{u}}^{-1}\right\} .
$$

Suppose $x_{0}, x_{1} \in W$ are equivalent coincidence points of $\phi$ and $\psi$. Thus, there is a path $p: I \rightarrow W$ such that $p(0)=x_{0}, p(1)=x_{1}$ and, for the splittings $\phi p=\left\{f_{1}, \ldots, f_{m}\right\}$ and $\psi p=\left\{g_{1}, \ldots, g_{n}\right\}$, we may assume that $f_{1}(0)=g_{1}(0), f_{1}(1)=g_{1}(1)$ and $f_{1}$ and $g_{1}$ are homotopic relative to the endpoints. Then $h_{1}: I \rightarrow X$ defined by $h_{1}(t)=f_{1}(t)\left(g_{1}(t)\right)^{-1}$ in the splitting of $\theta p$ is a loop in $X$ such that $h_{1}(0)=h_{1}(1)=e$. Now $e=h_{1}(0) \in \theta p(0)=\theta\left(x_{0}\right)$, so $x_{0} \in \theta^{-1}(e)$, and similarly, $e \in \theta\left(x_{1}\right)$, so $x_{0}$ and $x_{1}$ are roots of $\theta$ at $e$. Let $H: I \times I \rightarrow X$ be a homotopy such that $H(0, t)=f_{1}(t)$, 
$H(1, t)=g_{1}(t), H(s, 0)=f_{1}(0)=g_{1}(0)$ and $H(s, 1)=f_{1}(1)=g_{1}(1)$ for all $s, t \in I$. Define $K: I \times I \rightarrow X$ by $K(s, t)=H(s, t)\left(g_{1}(t)\right)^{-1}$. Then $K$ is a basepoint-preserving homotopy of the loop $h_{1}$ to the constant loop at $e$, which proves that $x_{0}$ and $x_{1}$ are equivalent roots of $\theta$ at $e$.

Conversely, suppose $x_{0}, x_{1} \in \theta^{-1}(e)$ are equivalent roots. Then there is a path $p: I \rightarrow W$ such that $p(0)=x_{0}, p(1)=x_{1}$ and, in the splitting $\theta p=\left\{h_{1}, \ldots, h_{u}\right\}$, the loop $h_{1}$ is homotopic to the constant loop at $e$. By Lemma 4.1, we may number the maps in the splittings of $\phi p$ and $\psi p$ so that $h_{1}(t)=f_{1}(t)\left(g_{1}(t)\right)^{-1}$. Then $h_{1}(0)=e$ implies $f_{1}(0)=g_{1}(0)$, where $f_{1}(0) \in \phi p(0)=\phi\left(x_{0}\right)$ and $g_{1}(0) \in \psi p(0)=\psi\left(x_{0}\right)$, so $\phi\left(x_{0}\right) \cap \psi\left(x_{0}\right) \neq \emptyset$ and $x_{0}$ is a coincidence point of $\phi$ and $\psi$; as is $x_{1}$, for the same reason. Let $K: I \times I \rightarrow X$ be a homotopy of $h_{1}$ to the constant loop, so $K(0, t)=$ $h_{1}(t)$ and $K(1, t)=K(s, 0)=K(s, 1)=e$. The homotopy $H: I \times$ $I \rightarrow X$ defined by $H(s, t)=K(s, t) g_{1}(t)$ shows that $f_{1}$ and $g_{1}$ are homotopic relative to the endpoints and thus, $x_{0}$ and $x_{1}$ are equivalent coincidence points of $\phi$ and $\psi$.

If $\phi: X \multimap X$ is an $n$-valued map and $\psi: X \rightarrow X$ is the identity map, then $\phi$ and $\psi$ determine the $n$-valued map $\theta: X \multimap X$, where $\theta(x)=\phi(x) x^{-1}$, that is, if $\phi(x)=\left\{x_{1}, \ldots, x_{n}\right\}$, then $\theta(x)=$ $\left\{x_{1} x^{-1}, \ldots, x_{n} x^{-1}\right\}$. The roots of $\theta$ at $e$ are thus the fixed points of $\phi$.

Theorem 4.2. Let $\phi: X \multimap X$ be an n-valued map of a compact connected Lie group $X$ with identity element $e$ and let $\theta: X \multimap X$ be the $n$-valued map determined by $\phi$ and $\psi$, where $\psi: X \rightarrow X$ is the identity map. Then the fixed point classes of $\phi$ are the root classes of $\theta$ at e. Moreover, a subset of $X$ is a geometrically essential fixed point class of $\phi$ if and only if it is an essential root class of $\theta$ and therefore $N(\theta, e)=\widehat{N}(\phi)$.

Proof. Let $\Phi: X \times I \multimap X$ be an $n$-valued homotopy such that $\phi_{0}=\phi$. Define $\Theta: X \times I \multimap X$ by $\Theta(x, t)=\Phi(x, t) x^{-1}$, that is, if $\Phi(x, t)=\left\{x_{1 t}, \ldots, x_{n t}\right\}$, then $\Theta(x, t)=\left\{x_{1 t} x^{-1}, \ldots, x_{n t} x^{-1}\right\}$. Let $\mathbf{F}_{0} \subseteq X \times\{0\}$ be a fixed point class of $\phi$. Then, by Lemmas 6.2 and 6.3 of [18], there is a unique fixed point class $\widehat{\mathbf{F}}$ of the fat homotopy $\widehat{\Phi}$ such that $\widehat{\mathbf{F}} \cap(X \times\{0\})=\mathbf{F}_{0}$. By Theorem 4 .1, with $W=X$ and $\psi(x)=x$, the set $\mathbf{F}_{0}$ is a root class of $\theta$ at $e$. Moreover, by Lemma 2.2 and Theorem 4.1, with $W=X \times I$ and $\Psi(x, t)=x$, the set $\widehat{\mathbf{F}}$ is the unique root class of $\Theta$ at $e$ containing $\mathbf{F}_{0}$.

If $\mathbf{F}_{0}$ is a geometrically inessential fixed point class of $\phi$, then there exists an $n$-valued homotopy $\Phi: X \times I \multimap X$ such that $\widehat{\mathbf{F}}$, the 
fixed point class of $\widehat{\Phi}$ containing $\mathbf{F}_{0}$, has the property $\widehat{\mathbf{F}} \cap(X \times\{1\})=$ $\emptyset$. Since, by Theorem 4.1 , the set $\widehat{\mathbf{F}}$ is also the unique root class of $\Theta$ at $e$ containing the root class $\mathbf{F}_{0}$ of $\theta$, we conclude that $\mathbf{F}_{0}$ is an inessential root class of $\theta$ at $e$.

Now suppose $\mathbf{F}_{0}$ is an inessential root class of $\theta$ at $e$. Then there exists an $n$-valued homotopy $\Theta: X \times I \multimap X$ such that $\theta_{0}=\theta$ and $\mathbf{F}$, the root class of $\Theta$ at $e$ containing $\mathbf{F}_{0}$, has the property $\mathbf{F} \cap(X \times\{1\})=\emptyset$. Define $\Phi: X \times I \multimap X$ as follows: if $\Theta(x, t)=$ $\left\{x_{1 t}, \ldots, x_{n t}\right\}$, then $\Phi(x, t)=\left\{x_{1 t} x, \ldots, x_{n t} x\right\}$, that is, $\Phi(x, t)=$ $\Theta(x, t) x$. Since $\Phi(x, t) x^{-1}=\Theta(x, t)$, Theorem 4.1 implies that $\widehat{\mathbf{F}}$ is the fixed point class of $\widehat{\Phi}$ that contains $\mathbf{F}_{0}$ and therefore, since $\widehat{\mathbf{F}} \cap(X \times\{1\})=\emptyset$, we conclude that $\mathbf{F}_{0}$ is a geometrically inessential fixed point class of $\phi$. We have proved that $N(\theta, e)=\widehat{N}(\phi)$.

By Proposition 3.1, Theorem 4.2 implies that $N(\theta, e)=N(\phi)$, the Nielsen fixed point number defined by Schirmer in [18], except, possibly, if $X$ is the 2-torus.

\section{$5(m, n)$-Valued pairs of maps of the circle}

We view the circle $S^{1}$ as the quotient group $S^{1}=\mathbb{R} / \mathbb{Z}$. Thus, we represent a point of $S^{1}$ by $s \in \mathbb{R}$, where $s_{0}$ and $s_{1}$ represent the same point of $S^{1}$ if and only if $s_{0}-s_{1} \in \mathbb{Z}$. For $\delta, \nu \in \mathbb{Z}$, where $\nu \geq 1$, define $\phi_{\nu, \delta}: S^{1} \multimap S^{1}$, the $\nu$-valued power map of degree $\delta$ [7], by

$$
\phi_{\nu, \delta}(s)=\left\{\frac{\delta s}{\nu}, \frac{\delta s}{\nu}+\frac{1}{\nu}, \ldots, \frac{\delta s}{\nu}+\frac{\nu-1}{\nu}\right\}
$$

for $0 \leq s<1$.

Proposition 5.1. The Nielsen root number at $1 \in S^{1}$ of the $\nu$ valued power map $\phi_{\nu, \delta}: S^{1} \multimap S^{1}$ is $N\left(\phi_{\nu, \delta}, 1\right)=|\delta|$.

Proof. Let $\phi=\phi_{\nu, \delta+\nu}$. Then for $\theta$ defined by $\theta(x)=\phi(x) x^{-1}$, we have $\theta=\phi_{\nu, \delta}$. Therefore, by Theorem 4.2 and by Theorem 4.1 of $[7]$,

$$
N\left(\phi_{\nu, \delta}, 1\right)=N(\theta, 1)=N(\phi)=|(\delta+\nu)-\nu|=|\delta| .
$$

Proposition 5.2. Let $a, b, m, n \in \mathbb{Z}$, where $1 \leq m \leq n$ and an $\neq$ bm. The pair of power maps $\phi_{m, a}, \phi_{n, b}: S^{1} \multimap S^{1}$ determine the $u$-valued power map $\theta=\phi_{u, \delta}$ of degree $\delta$, where

$$
u=\frac{m n}{<m, n>}, \quad \delta=\frac{a n-b m}{<m, n>}
$$


and $\langle m, n>$ denotes the greatest common divisor of $m$ and $n$.

Proof. The $(m, n)$-valued pair of power maps are defined by

$$
\begin{aligned}
\phi_{m, a}(s) & =\left\{\frac{a s}{m}, \frac{a s}{m}+\frac{1}{m}, \ldots, \frac{a s}{m}+\frac{m-1}{m}\right\} \\
\phi_{n, b}(s) & =\left\{\frac{b s}{n}, \frac{b s}{n}+\frac{1}{n}, \ldots, \frac{b s}{n}+\frac{n-1}{n}\right\}
\end{aligned}
$$

for $s \in[0,1)$. Let $\phi=\phi_{m, a}$ and $\psi=\phi_{n, b}$. Then

$$
\phi \psi^{-1}(s)=\left\{\left(\frac{a}{m}-\frac{b}{n}\right) s+\frac{j}{m}-\frac{k}{n}: j=0, \ldots, m-1 ; k=0, \ldots, n-1\right\} .
$$

Set

$$
u=\frac{m n}{<m, n>} .
$$

We will first show that

$$
\left(\frac{a}{m}-\frac{b}{n}\right) s+\frac{\ell}{u} \in \phi \psi^{-1}(s)
$$

for all $0 \leq \ell<u$ by proving that there exist $0 \leq j<m$ and $0 \leq k<n$ such that

$$
\left(\frac{j}{m}-\frac{k}{n}\right)-\frac{\ell}{u} \in \mathbb{Z}
$$

By Bézout's identity, we may write $\langle m, n\rangle=p m+q n$ for some $p, q \in \mathbb{Z}$. Choose $j \equiv \ell q(\bmod m)$ and $k \equiv-\ell p(\bmod n)$ so $j-\ell q=$ $\alpha m$ and $k+\ell p=\beta n$ for some $\alpha, \beta \in \mathbb{Z}$, then

$$
\begin{aligned}
\frac{j}{m}-\frac{k}{n} & =\frac{\ell q+\alpha m}{m}-\frac{-\ell p+\beta n}{n} \\
& =\frac{\ell q n+\alpha m n+\ell p m-\beta m n}{m n} \\
& =\frac{\ell<m, n>}{m n}+(\alpha-\beta) \\
& =\frac{\ell}{u}+(\alpha-\beta) .
\end{aligned}
$$

Now suppose $0 \leq j<m$ and $0 \leq k<n$ are given and let $0 \leq \ell<u$ such that

$$
\ell \equiv \frac{j n-k m}{<m, n>} \quad(\bmod u)
$$

Then

$$
\ell<m, n>-(j n-k m)=\alpha m n
$$


for some $\alpha \in \mathbb{Z}$ and thus

$$
\frac{\ell}{u}-\left(\frac{j}{m}-\frac{k}{n}\right)=\alpha .
$$

We have shown that

$$
\phi \psi^{-1}(s)=\left\{\left(\frac{a}{m}-\frac{b}{n}\right) s,\left(\frac{a}{m}-\frac{b}{n}\right) s+\frac{1}{u}, \ldots,\left(\frac{a}{m}-\frac{b}{n}\right) s+\frac{u-1}{u}\right\},
$$

which is the definition of the power map $\phi_{\nu, \delta}$, where

$$
\nu=u=\frac{m n}{<m, n>}
$$

and

$$
\frac{\delta}{\nu}=\frac{\delta}{u}=\frac{a}{m}-\frac{b}{n}
$$

Therefore,

$$
\delta=\frac{a n-b m}{<m, n>}
$$

and we set $\theta(s)=\phi_{u, \delta}(s)$.

As we remarked in Section 4, an $(m, n)$-valued pair of maps from a finite polyhedron to a Lie group may not determine a $u$-valued map. Moreover, as the following example demonstrates, even if the $(m, n)$-valued pair of maps does determine a $u$-valued map, this property will not necessarily be preserved under a homotopy of even one of the maps in the pair.

Example 5.1. By Proposition 5.2, the pair of power maps $\phi_{2,1}, \phi_{3,1}$ determine the power map $\phi_{6,1}$. Let $f(s)=s(1-s)$ and define $\phi: S^{1} \multimap S^{1}=\mathbb{R} / \mathbb{Z}$ by

$$
\phi(s)=\left\{\frac{s}{2}, \frac{s}{2}+\frac{1}{2}-f(s)\right\},
$$

which is a 2-valued map because $0 \leq f(s) \leq \frac{1}{4}$ for $0 \leq s<1$, and it is 2-valued homotopic to $\phi_{2,1}$. Let $\psi=\phi_{3,1}$, then

$\phi \psi^{-1}(s)=\left\{\frac{s}{6}, \frac{s}{6}+\frac{1}{6}-f(s), \frac{s}{6}+\frac{1}{3}, \frac{s}{6}+\frac{1}{2}-f(s), \frac{s}{6}+\frac{2}{3}, \frac{s}{6}+\frac{5}{6}-f(s)\right\}$,

so $\phi \psi^{-1}(0)$ takes on six distinct values. But $f\left(\frac{\sqrt{3}+1}{2 \sqrt{3}}\right)=\frac{1}{6}$, so there are only three distinct values

$$
\phi \psi^{-1}\left(\frac{\sqrt{3}+1}{2 \sqrt{3}}\right)=\left\{\frac{\sqrt{3}+1}{12 \sqrt{3}}, \frac{5 \sqrt{3}+1}{12 \sqrt{3}}, \frac{9 \sqrt{3}+1}{12 \sqrt{3}}\right\} .
$$

Therefore, the $(2,3)$-valued pair of maps $\phi, \psi$ does not determine a u-valued map. 
Proposition 5.3. If an $\neq b m$, then the number of coincidence points of the power maps $\phi_{m, a}$ and $\phi_{n, b}$ is

$$
\# \operatorname{Coin}\left(\phi_{m, a}: \phi_{n, b}\right)=\frac{|a n-b m|}{<m, n>}
$$

Proof. By Proposition 5.2, the power maps $\phi_{m, a}$ and $\phi_{n, b}$ determine the power map $\theta$ defined by

$$
\theta(s)=\left\{\left(\frac{a}{m}-\frac{b}{n}\right) s+\frac{j}{u} ; j=0,1, \ldots, u-1\right\},
$$

where $u=\frac{m n}{<m, n>}$. Since a coincidence point of the power maps is a root at 0 of the power map that they determine, then $s \in$ $\operatorname{Coin}\left(\phi_{m, a}: \phi_{n, b}\right)$ if and only if $0 \in \theta(s)$, that is, for some $j \in$ $\{0,1, \ldots, u-1\}$,

$$
\left(\frac{a}{m}-\frac{b}{n}\right) s+\frac{j}{u}=0
$$

SO

$$
\frac{(a n-b m)}{m n} s=-\frac{<m, n>}{m n} j
$$

and thus

$$
\frac{(a n-b m)}{<m, n>} s \in \mathbb{Z}
$$

Since the number of solutions $s$ in the interval $0 \leq s<1$ is $\frac{|a n-b m|}{<m, n>}$, we conclude that

$$
\# \operatorname{Coin}\left(\phi_{m, a}: \phi_{n, b}\right)=\# \theta^{-1}(0)=\frac{|a n-b m|}{<m, n>} .
$$

The degree $\operatorname{deg}(\phi)$ of an $n$-valued map $\phi: S^{1} \multimap S^{1}$ of the circle is defined in [7] in terms of the lift of the map to $\mathbb{R}$. Alternatively, using the fact from page 56 of [8] that an $n$-valued map $\phi$ of the circle induces a (single-valued) endomorphism $\phi_{*}: H_{1}\left(S^{1}\right) \rightarrow H_{1}\left(S^{1}\right) \cong \mathbb{Z}$, then $\operatorname{deg}(\phi)$ may be defined as for single-valued maps.

Theorem 5.1. Let $\phi, \psi: S^{1} \multimap S^{1}$ be an $(m, n)$-valued pair of maps, where $\operatorname{deg}(\phi)=a, \operatorname{deg}(\psi)=b$. Then

$$
N(\phi: \psi)=\frac{|a n-b m|}{<m, n>} .
$$

Proof. By Theorem 3.1 of [7], $\phi$ is homotopic to the power map $\phi_{m, a}$ and $\psi$ is homotopic to $\phi_{n, b}$, so $N(\phi: \psi)=N\left(\phi_{m, a}: \phi_{n, b}\right)$ by Theorem 2.1. 
Suppose $a n=b m$. Given an $n$-valued $\operatorname{map} \phi: S^{1} \multimap S^{1}=\mathbb{R} / \mathbb{Z}$ and $\epsilon>0$, define $\phi^{\epsilon}: S^{1} \multimap S^{1}$ as follows: if $\phi(s)=\left\{s_{1}, \ldots, s_{n}\right\}$, then $\phi^{\epsilon}(s)=\left\{s_{1}+\epsilon, \ldots, s_{n}+\epsilon\right\}$. Then $\phi^{\epsilon}$ is $n$-valued homotopic to $\phi$. Since $a n=b m$, then for $\epsilon$ sufficiently small, $\operatorname{Coin}\left(\phi_{m, a}: \phi_{n, b}^{\epsilon}\right)=\emptyset$ and therefore $N(\phi: \psi)=0$ in this case.

For the rest of the proof, we assume that $a n \neq b m$. Let $\theta$ be the power map determined in Proposition 5.2 by $\phi_{m, a}$ and $\phi_{n, b}$. By Propositions 5.1 and 5.2,

$$
N(\theta, 1)=|\operatorname{deg}(\theta)|=\frac{|a n-b m|}{<m, n>},
$$

which is the number of roots of $\theta$ at 1 since, by Proposition 5.3, that is the number of coincidences of $\phi_{m, a}$ and $\phi_{n, b}$. Thus, by Theorem 4.1 , each root of $\theta$ at 1 is both a root class and a coincidence class. The local degrees ([11], Definition 4.2, page 267) of the roots of the power map $\theta$ at 1 are either all +1 or all -1 . Let $z_{0} \in S^{1}$ be a root of $\theta$ at 1 and let $U$ be a simply-connected neighborhood of $z_{0}$ that contains no other root. Let $v: U \rightarrow S^{1}$ be inclusion, so there are splittings $\phi_{m, a} v=\left\{f_{1}, \ldots, f_{m}\right\}$ and $\phi_{n, b} v=\left\{g_{1}, \ldots, g_{n}\right\}$ and thus, by Lemma $4.1, \theta v=\left\{h_{1}, \ldots, h_{u}\right\}$ where $h_{i}=f_{j_{i}} g_{k_{i}}^{-1}$. We renumber the maps in the splittings so that $f_{1} g_{1}^{-1}\left(z_{0}\right)=1$ and thus, the local degree at $z_{0}$ is that of $f_{1} g_{1}^{-1}$. Let $e^{i 2 \pi s_{0}}=z_{0}$, where $0 \leq s_{0}<1$. Then the local degree of $f_{1} g_{1}^{-1}$ at $z_{0}$ equals the degree of $f_{1}-g_{1}$ at $s_{0}$. By definition, the coincidence index in this setting is also the degree of $f_{1}-g_{1}$ at $s_{0}$ ([16], page 23), so the coincidence index of $f_{1}$ and $g_{1}$ at $z_{0}$ is nonzero. Let $\Phi, \Psi: S^{1} \times I \multimap S^{1}$ be an $(m, n)$-valued pair of homotopies such that $\phi_{0}=\phi_{m, a}$ and $\psi_{0}=\phi_{n, b}$. Let $\mathbf{C}$ be the coincidence class of $\Phi$ and $\Psi$ that contains the coincidence class $z_{0}$ and let $\mathbf{C}_{t}=\mathbf{C} \cap\left(S^{1} \times\{t\}\right)$. The hypothesis $a n \neq b m$ implies that $\operatorname{Coin}\left(\phi_{t}, \psi_{t}\right)$ is a proper subset of $S^{1}$ for all $t$. Therefore, $\operatorname{Coin}\left(\phi_{t}, \psi_{t}\right)$ is contained in a simply-connected subset on which $\phi_{t}$ and $\psi_{t}$ are split, so we may apply the coincidence theory of single-valued maps. By the homotopy property of the coincidence index ([20], Lemma 6.8 , page 180), the coincidence index of $\mathbf{C}_{t}$ is nonzero. In particular, Lemma 6.2 of $[20]$ therefore implies that $\mathbf{C}_{1} \neq \emptyset$ and thus, the coincidence class $z_{0}$ is essential. We have demonstrated that each coincidence point of $\phi_{m, a}$ and $\phi_{n, b}$ is an essential coincidence class. Therefore

$$
N(\phi: \psi)=N\left(\phi_{m, a}: \phi_{n, b}\right)=\frac{|a n-b m|}{<m, n>} .
$$


¿From Theorem 5.1 and Proposition 5.3 we have

Corollary 5.1. If $\phi, \psi: S^{1} \multimap S^{1}$ is an $(m, n)$-valued pair of maps where $\operatorname{deg}(\phi)=a, \operatorname{deg}(\psi)=b$, then

$$
N(\phi: \psi)=M C(\phi: \psi)=\frac{|a n-b m|}{<m, n>} .
$$

\section{References}

[1] Better, J., Equivariant Nielsen fixed point theory for n-valued maps, Topology Appl., 157, 1804 - 1814 (2010).

[2] Better, J. A Wecken theorem for n-valued maps, Topology Appl., 159, 3707 - 3715 (2012).

[3] Brooks, R. On the sharpness of the $\Delta_{2}$ and $\Delta_{1}$ Nielsen numbers, J. reine ang. Math., 259, 101 - 108 (1973).

[4] Brooks, R. Nielsen root theory, Handbook of Topological Fixed Point Theory, Springer, 2005, 375 - 432.

[5] Brooks, R. and Brown, R. A lower bound for the $\Delta$-Nielsen number, Trans. Amer. Math. Soc. 143, 555 - 564 (1969).

[6] Brown, M., Nielsen root theory of n-valued maps, preprint.

[7] Brown, R., Fixed points of n-valued multimaps of the circle, Bull. Polish Acad. Sci. Math., 54, 153 - 162 (2006).

[8] Brown, R. The Lefschetz number of an n-valued multimap, JP Journal of Fixed Point Theory Appl., 2, 53 - 60 (2007).

[9] Brown, R. Nielsen numbers of n-valued fiber maps, J. Fixed Point Theory Appl., 4, 183 - 201 (2008).

[10] Brown, R. and Lin, J. Coincidences of projections and linear n-valued maps of tori, Topology Appl., 157, 1990 - 1998 (2010).

[11] Dold, A., Lectures on Algebraic Topology, 2nd edition, Springer, 1980.

[12] Gabor, G., Gorniewicz, L. and Slosarski, M. Generalized topological essentiality and coincidence points of multivalued maps, Set-Valued Var. Anal., 17, 1 - 19 (2009).

[13] Goncalves, D., Coincidence Theory, Handbook of Topological Fixed Point Theory, Springer, 2005, 3 - 42. 
[14] Jiang, B., Fixed points and braids, II, Math. Ann., 272, 249 256 (1985).

[15] Jiang, B., A primer of Nielsen fixed point theory, Handbook of Topological Fixed Point Theory, Springer, 2005, 617 - 645.

[16] Schirmer, H., Mindestzahlen von Koinzidenzpunkten, J. reine ang. Math. 194, 21 - 39 (1955).

[17] Schirmer, H., Fix-finite approximations of $n$-valued multifunctions, Fund. Math. 121, 73 - 80 (1984).

[18] Schirmer, H., An index and Nielsen number for n-valued multifunctions, Fund. Math. 124, 207 - 219 (1984).

[19] Schirmer, H., A minimum theorem for n-valued multifunctions, Fund. Math. 126, 83 - 92 (1985).

[20] Vick, J. Homology Theory: An Introduction to Algebraic Topology, Academic Press, 1973.

[21] Wlodarczyk, K. and Klim, D., Fixed point and coincidence theorems for set-valued maps with nonconvex or noncompact domains in topological vector spaces, Abst. Appl. Anal. 2003, 1 - 18. 